\title{
Piezoresistive behaviour of graphene nanoplatelet (GNP)/PMMA spray coated sensors on a polymer matrix composite beam
}

\author{
D. Sethy ${ }^{1}$, S. Makireddi ${ }^{2}, F$. V. Varghese ${ }^{3}$, K. Balasubramaniam ${ }^{1 *}$ \\ ${ }^{1}$ Center for Non-destructive Evaluation, Indian Institute of Technology Madras, 600036 Chennai, India \\ ${ }^{2}$ Aircraft Research and Design Centre, Hindustan Aeronautics Limited, 560037 Bengaluru, India \\ ${ }^{3}$ Defence Bioengineering and Electromedical Laboratory, 560093 Bengaluru, India
}

Received 23 March 2019; accepted in revised form 18 June 2019

\begin{abstract}
Graphene nanoplatelet (GNP)/poly(methyl methacrylate) (PMMA) nanocomposite solution was spray coated on a glass fibre reinforced polymer composite (GFRP) beam with different initial electrical resistance $\left(R_{0}\right)$. Scotch tape erosion method was used to tailor the $R_{0}$ of the sensors. Beams and the sensors were characterized by computed tomography (CT) and scanning electron microscopy (SEM) respectively. The piezoresistive behaviour of these sensors was evaluated in monotonic, step and cyclic loading conditions. These spray coated sensors offered good sensitivity (38.5 times) as compared to a strain gauge. A gauge factor (GF) of $55 \pm 0.5,70 \pm 2$, and $77 \pm 1$ was obtained for $R_{0}$ of 1,7 and $21 \mathrm{k} \Omega$ GNP layers, respectively. Sensors showed good response and stability under the step and cyclic loading conditions. The ease in the process of application coupled with good sensitivity demonstrates that the GNP/PMMA spray coated sensor can be a potential candidate for the futuristic multi-functional materials for structural health monitoring.
\end{abstract}

Keywords: polymer composites, nanomaterials, graphene, coating, piezoresistivity

\section{Introduction}

Polymer matrix composites (PMCs) are being used in the aerospace industry owing to their good stiffness, high strength to weight ratio, and resistance to fatigue and corrosion. However, these structures are vulnerable to impact-induced damages. These damages are barely visible on the impact surface due to the anisotropic nature of this material while they may be significant in other areas [1]. It is generally uneconomical to inspect these structures by regular non-destructive testing (NDT) methods and hence smart sensors are being developed for the health monitoring of these structures [2]. The advances in the field of nano-scale conductive fillers such as carbon nanotubes (CNT) and graphene have led to the development of electrically conductive smart PMCs
[3-4]. Apart from the conductivity, these nano-fillers are reported to exhibit properties such as piezoresistivity and can be used either to impart smartness in the host structures or as sensors to monitor strain in the structural health monitoring (SHM) [5-7]. The electrical resistance of such materials changes when they experience strain and deformation, this phenomenon is defined as piezoresistive effect and provides an easy and direct energy/signal transduction between the mechanical and the electrical domains [8]. Recently, graphene, a 2D nano-filler, has attracted great interest due to its unique properties such as extremely high thermal and electrical conductivity, mechanical strength and piezoresistivity [9-11]. These outstanding properties of graphene have led to the development of strain sensors for SHM [12-14]. 
Three different approaches have been proposed for strain monitoring of structures using graphene. First one is to develop a flexible nanocomposite film which can be glued to the structure in order to measure the strain as it is done at present with metal foil strain gauges. In the second approach, graphene nano-fillers are embedded in the matrix or attached to the reinforcing fibres to prepare a composite lamina/laminate. Another approach is to apply the graphenebased smart paint directly on the structure and use it as a strain sensor. The sensitivity of these sensors is measured in terms of gauge factor $(G F)$ which is the ratio of change in the electrical resistance $(\Delta R / R)$ of the sensor to strain $(\varepsilon)$.

Graphene or various other forms of graphitic materials such as GO (graphene oxide), reduced GO, functionalized GO, GNP etc. incorporated in a wide variety of polymer matrices are being used to develop flexible nanocomposite film sensors having GF ranging from 0.55 to 300 [15-18]. But this method involves complex processes of dispersion of graphene in the viscous polymer matrices and casting the film by avoiding the re-agglomeration during the film formation stage [19-21].

Using the second method, a GF of 56.7 and 300 were reported for GNPs embedded in the epoxy matrix [22] and polyvinyl ester [23] respectively and observed that the response of the sensor is affected by the intrinsic properties of the polymer.

The spray or paint technique enables the pre-dispersed graphene solution to be painted directly and uniformly using compressed air/Nitrogen at desired locations on the structure [20]. Tamburrano et al. [23] spray deposited monolayer graphene on a polycarbonate beam, investigated the sensor performance through static electro-mechanical tests and reported a GF of 30 at 50 micro strains. The researchers at Italian Aerospace Research Center working for a European Union funded project, called 'Smart Intelligent Aircraft Structures' (SARISTU) developed an array of eight sensors by spray coating graphene on a carbon fibre reinforced composite (CFRC) structure and reported an engineering GF of 200 for strains up to $0.14 \%$ [24].

Although high sensitivities were reported using GNP based nanocomposite film sensors, not much work has been done on the GNP coatings deposited directly on the surface of the structure. Particularly, the effect of GNP density on the sensitivity and their performance under different mechanical loading conditions are not explored. In the present work, a spray deposited GNP/PMMA smart layer on a glass fiber reinforced polymer (GFRP) composite specimen is used as a sensor to monitor the strain and studied its performance under various practical loading conditions.

\section{Experimental}

Quasi-isotropic, 8-layer (0/+45/90/-45/0/-45/90/+45) GFRP composite specimen of size $250 \mathrm{~mm} \times 25 \mathrm{~mm}$ were prepared via vacuum assisted resin transfer moulding (VARTM) process with a thickness of $3 \pm 0.1 \mathrm{~mm}$. Epoxy based RIM 135 and RIMH 134 were used as a matrix and hardener, respectively. GNPs (thickness $<3 \mathrm{~nm}$; diameter $<10 \mu \mathrm{m}$ ) prepared by low-temperature physical exfoliation were obtained generously from GRAPHENE LAB Ltd, London, UK and used without any further treatment. Poly(methyl methacrylate) (PMMA) (average $M_{\mathrm{W}}$ $120000 \mathrm{~g} / \mathrm{mol}$ ) was purchased from Sigma-Aldrich. Tetrahydrofuran (THF)-AR was used as a solvent. WIREGLUE was obtained from Idolon Technologies, MA, US.

GNPs $(20 \mathrm{mg}$ ) were added to $80 \mathrm{ml}$ THF and ultrasonicated using an ELMA Transonic Ti-H-5 bath sonicator, $135 \mathrm{kHz}$ at $80 \%$ for $180 \mathrm{~min}$. A stock solution of PMMA/THF was separately prepared by dissolving $0.2 \mathrm{mg}$ of PMMA in $20 \mathrm{ml}$ THF, using a magnetic stirrer. $5 \mathrm{ml}$ of this stock solution was added to the GNP-THF dispersion and ultrasonicated for another $60 \mathrm{~min}$. The GNP/PMMA solution was poured into a spray gun and was sprayed on to the composite beam specimen on a marked area (30 $\mathrm{mm} \times 20 \mathrm{~mm}$ ) using nitrogen gas. Thus, formed GNP/PMMA sensor was allowed to dry for 48 hours at room temperature. Lead wires were attached at both ends of the sensor using wire glue. The resistance of the sensor was adjusted roughly to $1 \mathrm{k} \Omega$ by etching the smart layer several times using scotch tape. The steps involved in the preparation of GNP sensors over GFRP specimen are shown in Figure 1. In a similar manner, 7 and $21 \mathrm{k} \Omega$ layers were also prepared.

Another GFRP beam, glued with a standard strain gauge HBM1-LY41-6/350 (obtained from HBM India), having an initial resistance of $0.35 \mathrm{k} \Omega$ was used for the comparison of the results. Piezoresistive behaviour of the sensor was determined by straining the beam in the Instron Universal Testing Machine while monitoring the change in the resistance of the sensor continuously with Keithley 2450 source meter. 


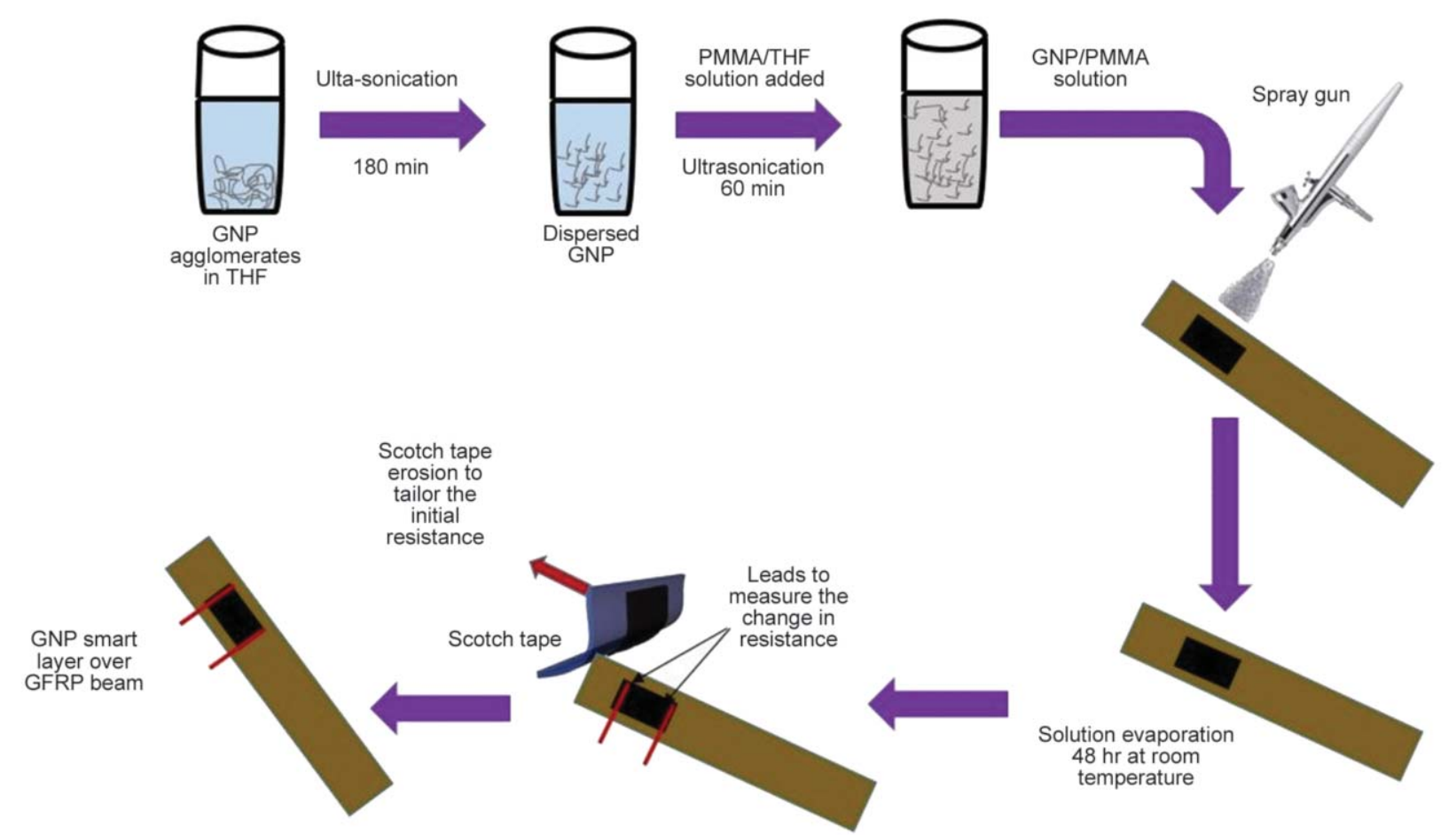

Figure 1. Method of preparation of GNP smart layer on GFRP composite beams (A small area of $30 \mathrm{~mm} \times 20 \mathrm{~mm}$ size was chosen rather than coating complete surface of the beam).

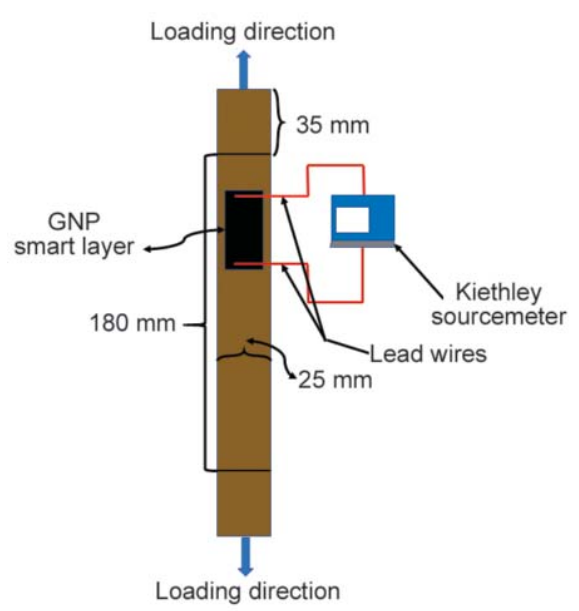

Figure 2. Schematic of the experimental set up to measure the piezoresistive behaviour of the smart layers.

The schematic and the experimental setup to measure the piezoresistive behaviour of the sensors are shown in Figure 2.

\section{Characterization}

\subsection{Computed tomography (CT) scan of GFRP composite}

Bare GFRP composite samples were scanned in the Phoenix Vtomex industrial high-resolution $\mathrm{CT}$ and $\mathrm{X}$-ray system, equipped with $240 \mathrm{kV} / 320 \mathrm{~W}$ highpower micro focus X-ray tube. CT scan is used to inspect the layers in a composite. Figure 3 a shows the topography of the surface which is rough and we can find the crest (bright lines) and valley (dull lines) sort of surface. Figure $3 b$ and $3 c$ show the orientation of the fibers in the composite.

\subsection{Scanning electron microscopy (SEM)}

GNP spray coated sensors were subjected to a low magnification SEM (INSPECT F-50) to assess the morphology of the layer, at different resolutions and shown in Figure 4. The channel-like morphology belongs to the naked beam surface, resembling ridges and trenches impinged with the GNPs. In Figures $4 \mathrm{a}$ and $4 \mathrm{~b}$, these platelets are lumped together and oriented randomly because of several layers of spray deposition $\left(R_{0} \sim 1 \mathrm{k} \Omega\right)$. A maximum number of particles are found in the trenches and a very few GNPs are stuck to the ridges. Figures $4 \mathrm{c}$ and $4 \mathrm{~d}$ show the morphology and inter-particle distance in the smart layer after several times of scotch tape erosion $\left(R_{0} \sim\right.$ $21 \mathrm{k} \Omega$ ). The number density of the GNPs is observed to be more in the $1 \mathrm{k} \Omega$ sample as compared to the $21 \mathrm{k} \Omega$ sample.

\section{Results and discussion}

Piezoresistive behaviour of these sensors was tested under three different tensile loading conditions i.e. monotonic uniaxial, step and cyclic and the electrical resistance response of the layer was measured and expressed as $\% \Delta R / R$. 


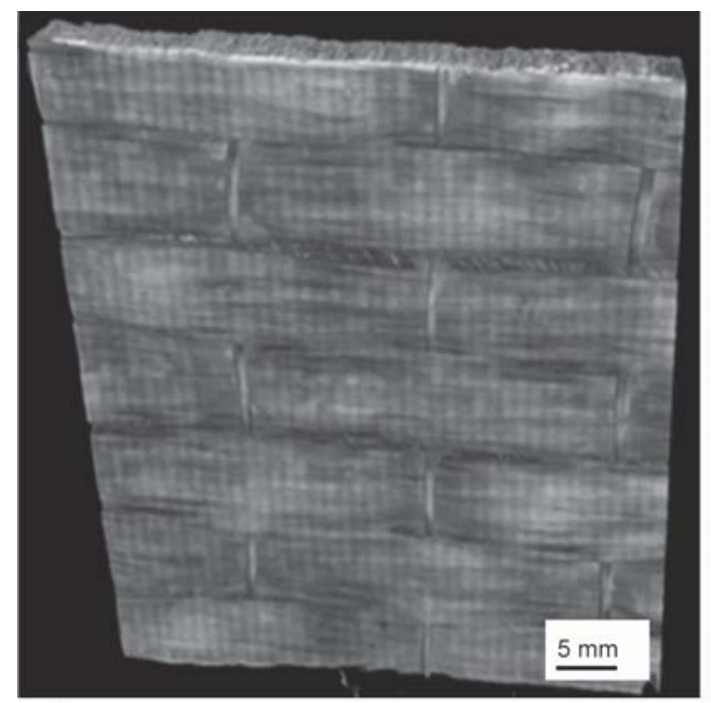

a)

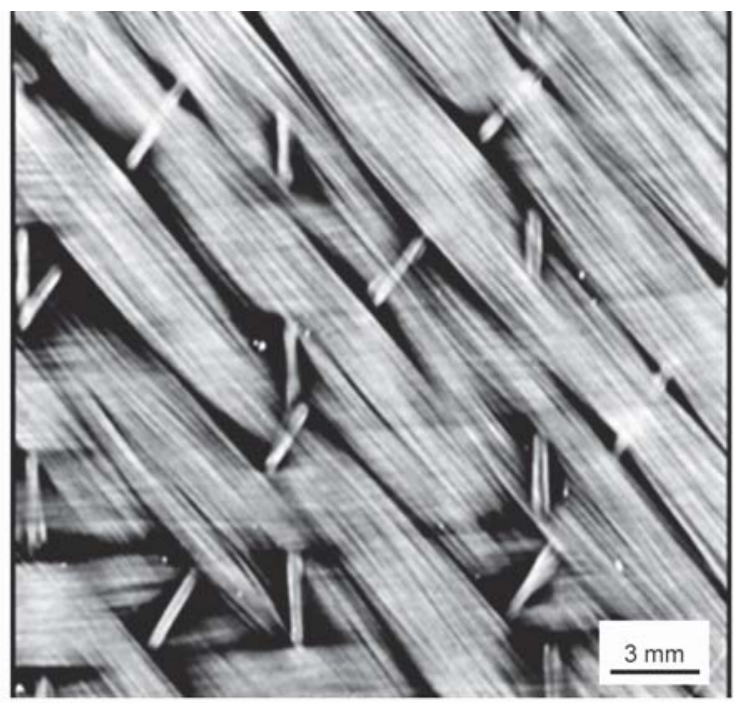

b)

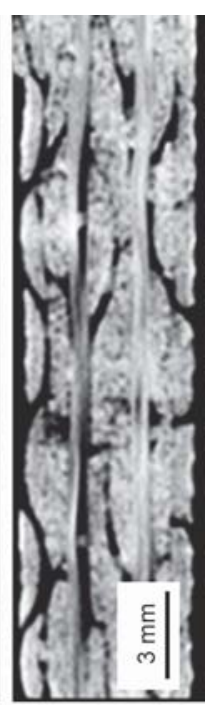

Figure 3. Computed tomography (CT) scanning of GFRP samples a) surface of the specimen, b) orientation of the GFRP fibers inside the composite and c) side view.

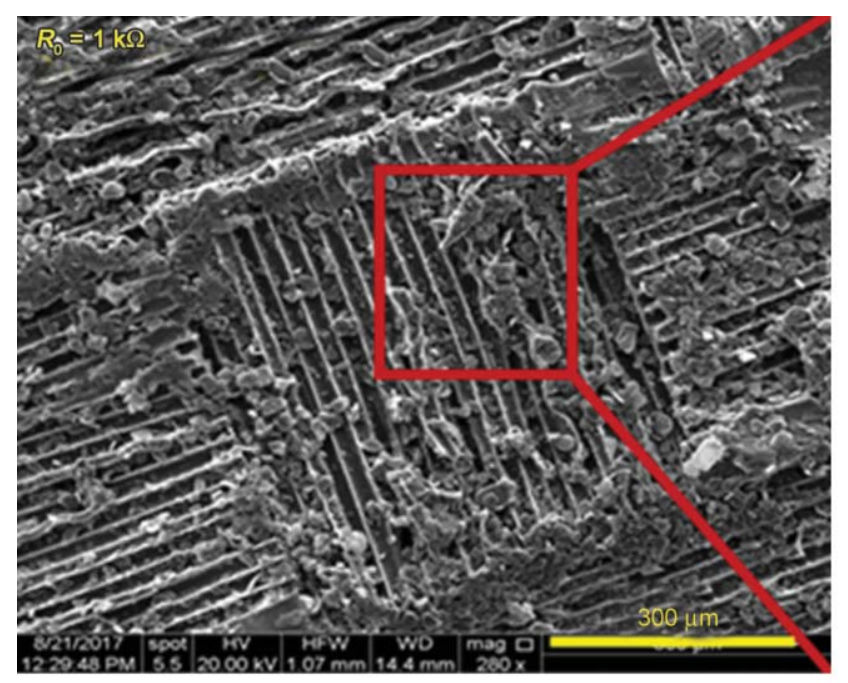

a)

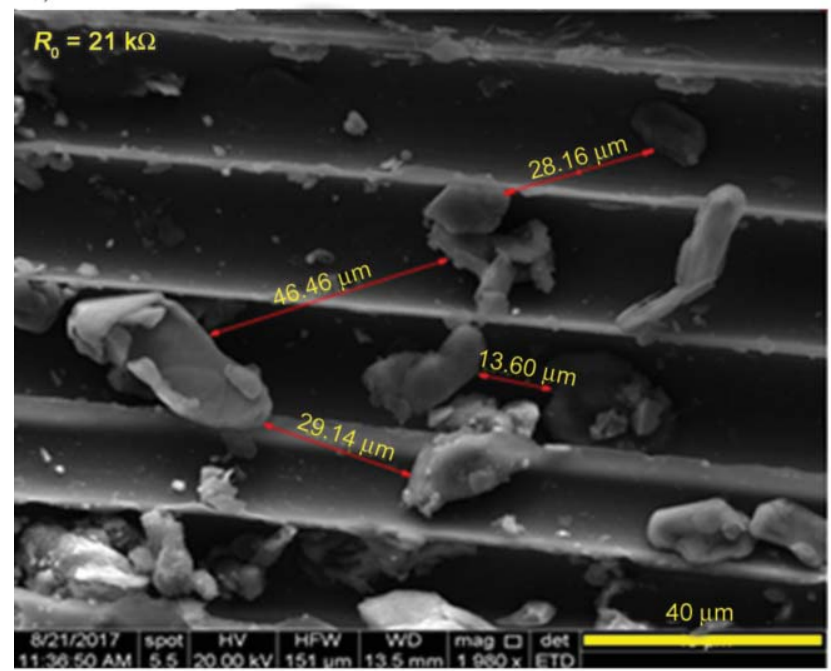

c)

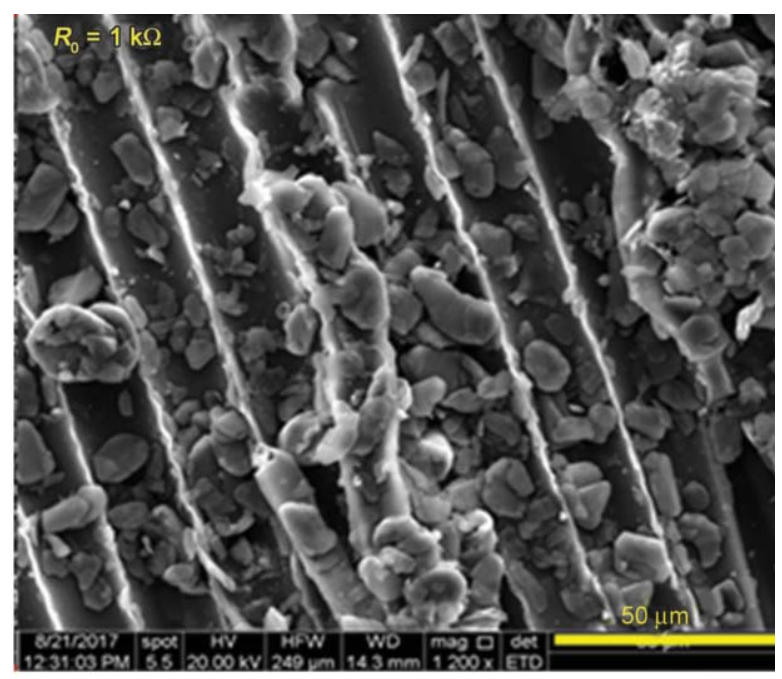

b)

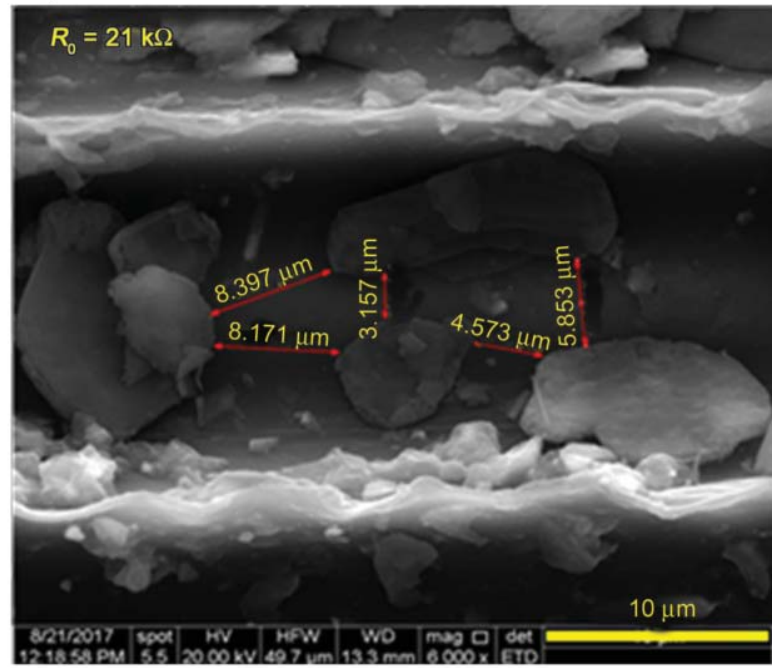

d)

Figure 4. SEM images of the GNP layer on GFRP at different resolutions; a) GNPs sprayed upon composite which got stuck to the surface of the composite ridges and valleys, b) zoomed image of the rectangular portion, c) sample subjected to scotch tape erosion several times showing less number of GNPs and the inter-particle distance and d) GNPs stuck on the surface of the beam. 


\subsection{Monotonic uniaxial loading}

Monotonic uniaxial testing was done to assess the sensitivity of the sensor with different initial resistance $\left(R_{0}\right)$ and to calculate the GF. The load on the specimen was uniaxially applied at a rate of $2 \mathrm{~mm} / \mathrm{min}$. Figure 5 a shows the sensitivity of the standard strain gauge where a GF of 1.6 was measured from the slope of the curve. Figure $5 \mathrm{~b}$ shows the change in the resistance $(\% \Delta R / R)$ of the smart layers with strain. While the change in resistance of all the beams is linearly increased with strain, the beam with high initial resistance $(21 \mathrm{k} \Omega)$ smart layer has shown better sensitivity to strain. An approximate
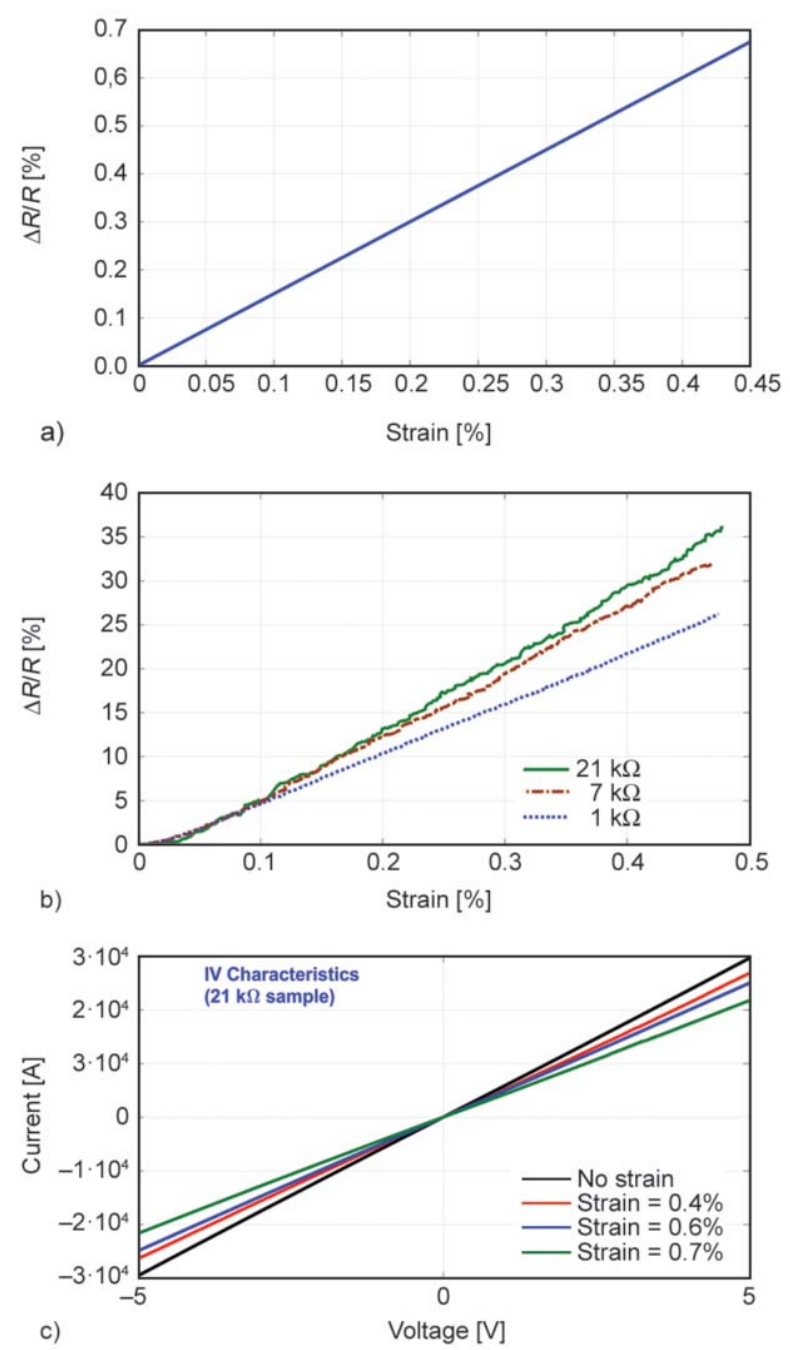

Figure 5. Change in the resistance of the standard strain gauge - HBM1-LY41-6/350 $\left(R_{0}=0.35 \mathrm{k} \Omega\right.$; measured GF is $\sim 1.6)$ b) change in the resistance $(\Delta R / R)$ of the GFRP beams with strain for different initial resistance $\left(R_{0}\right)$; the GFRP beams with $R_{0}$ of 1,7 , and $21 \mathrm{k} \Omega$ have shown an approximate GF of $55 \pm 0.5,70 \pm 2$, and $77 \pm 1$, respectively. c) IV characteristics of GNP layer $\left(R_{0}=21 \mathrm{k} \Omega\right)$ with and without strain.
GF of $77 \pm 1$ is obtained from the slope of this curve by best fit from data of three tests. As the $R_{0}$ is decreased, the slope of the curve is decreased, indicating a decrease in the sensitivity. The sensors with $R_{0}$ of $1 \mathrm{k}, 7 \mathrm{k} \Omega$ have shown a GF of $55 \pm 0.5,70 \pm 2$, respectively. Not much difference in the piezoresistive sensitivity is observed between 7 and $21 \mathrm{k} \Omega$ samples. VI characteristics of the sensors were investigated under different strains to check the linearity (Figure $5 \mathrm{c}$ ). As seen from the figure these sensors retain their ohmic behaviour and the resistance of the sensor is increased with the strain which is caused by the increased tunnelling distance among GNPs with the strain.

Researchers explained the piezoresistive behaviour of the nanocomposites through the tunnelling effect. When the gap between the adjacent conductive particles is small enough, the conductivity takes place between the particles through quantum mechanical tunnelling. Only the probability of this effect reduces with the increase in the inter-particle distance [25, $26]$. In the $1 \mathrm{k} \Omega$ layer, there is a number of GNPs in the network with a smaller inter flake distance as compared to $21 \mathrm{k} \Omega$. When such a beam is strained, even a few GNP networks have broken due to strain, several alternative conductive paths are available for the electrons to conduct, and hence a smaller change in the resistance is observed. For $21 \mathrm{k} \Omega$ layer, with less number of particles and an optimum distance between the particles favouring for the tunneling of electrons, a greater change in the resistance can be observed when such networks have broken due to strain $[18,20]$.

\subsection{Stepped loading/unloading}

Stepped tensile loading was performed to study the performance of GNP/PMMA sensors under practical loading conditions such as increasing the load, holding it for a certain time and then increase or decrease the load. Here the tensile load was increased from 0 to $5 \mathrm{kN}$ in three steps, holding for $10 \mathrm{sec}$ at each load (Figure 6a). In a similar manner, the load on the specimen was decreased to zero. During the entire course of loading and unloading the change in the resistance of the standard strain gauge and GNP layer was monitored and plotted in Figure $6 \mathrm{~b}$ and $6 \mathrm{c}$, respectively. It can be observed that the change in resistance of the sensor is increased with the increase in the load and was observed to be constant when the load is held constant. Here the time response of the 


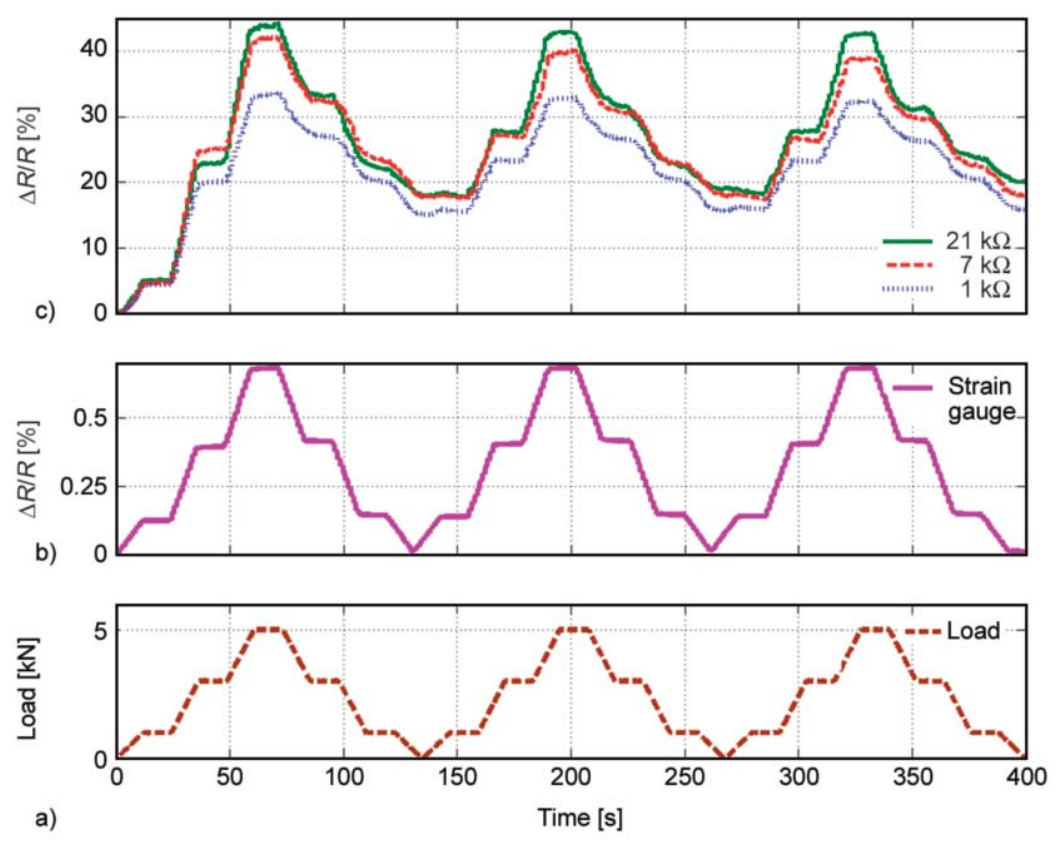

Figure 6. a) Loading/unloading cycles of the GFRP specimen with time. b) Change in the resistance of the strain gauge and c) Change in the resistanceof the GFRP specimen with various initial resistances $\left(R_{0}\right)$ GNP layers (about $40 \%$ of the total piezoresistivity observed during first loading is not recovered after unloading and shift in the base line for subsequent loading/unloading cycles can be observed).

sensor $(\% \Delta R / R)$ is matching with the time of loading and there is no observable time lag between the input (load) and the response $(\% \Delta R / R)$. But in the first cycle, during the unloading phase, even when the GFRP specimen is completely unloaded, the resistance response did not reach the zero base line. Instead, the base line got shifted to a new level of about $15 \%$ for $1 \mathrm{k} \Omega$ sensor. As the $R_{0}$ increased, this base line shift is also increased. For $21 \mathrm{k} \Omega$ sample, this shift is observed to be around $18-20 \%$. This shift is found to be stable for further loading and unloading cycles. This behaviour of the sensor can be attributed to the irreversible re-orientation of GNPs with loading and unloading which resulted in the permanent set in the network.

\subsection{Cyclic loading}

The samples were subjected to tension-tension fatigue cycles at a $0.01 \mathrm{~Hz}$ frequency in load control mode of $1 \mathrm{kN}$ (Figure 7a) in order to test the cyclic behaviour of the sensors. Figure $7 \mathrm{~b}$ shows the response of a standard strain gauge. Figure $7 \mathrm{c}$ shows the normalized resistance for all smart layers with different $R_{0}$ which is observed to be increased with an increase in the load and with an increase in $R_{0}$, without any time delay in the response. Here also the base line shift can be observed during the first cycle which is almost constant in the further cycles. It is interesting to see that this shift is directly proportional to the sensitivity i.e. high sensitivity sample has shown a greater shift. This may be because the GNPs are loosely stuck to the surface of the GFRP and are reoriented thus causing the change in the initial resistance of the layer. However, this base line shifting behaviour under cyclic loading was also observed in the sensors made from multi-walled carbon nanotubes (MWCNT)/Ecoflex spray coated films [27], GNP/PMMA solution cast films [28] and screen printed silver-coated polystyrene spheres/poly dimethyl siloxane (PDMS) films [29].

\section{Conclusions}

We report the piezoresistive behaviour of spray coated GNP sensors on the GFRP composite specimen, under three different loading conditions. The scotch tape erosion method is used to tailor the initial resistance. A GF of $55 \pm 0.5,70 \pm 2$, and $77 \pm 1$ was obtained for $R_{0}$ of 1,7 and $21 \mathrm{k} \Omega$, respectively. The results show that the smart layers exhibit a significantly higher sensitivity than the commercial strain gauge. The GF is found to be dependent upon the GNP density on the surface. These spray coated sensors suffer from some initial drift of baseline resistivity during the cyclic loading. Although the base line shift behaviour is not sought in practical applications, the high sensitivity, ease in the method of application 

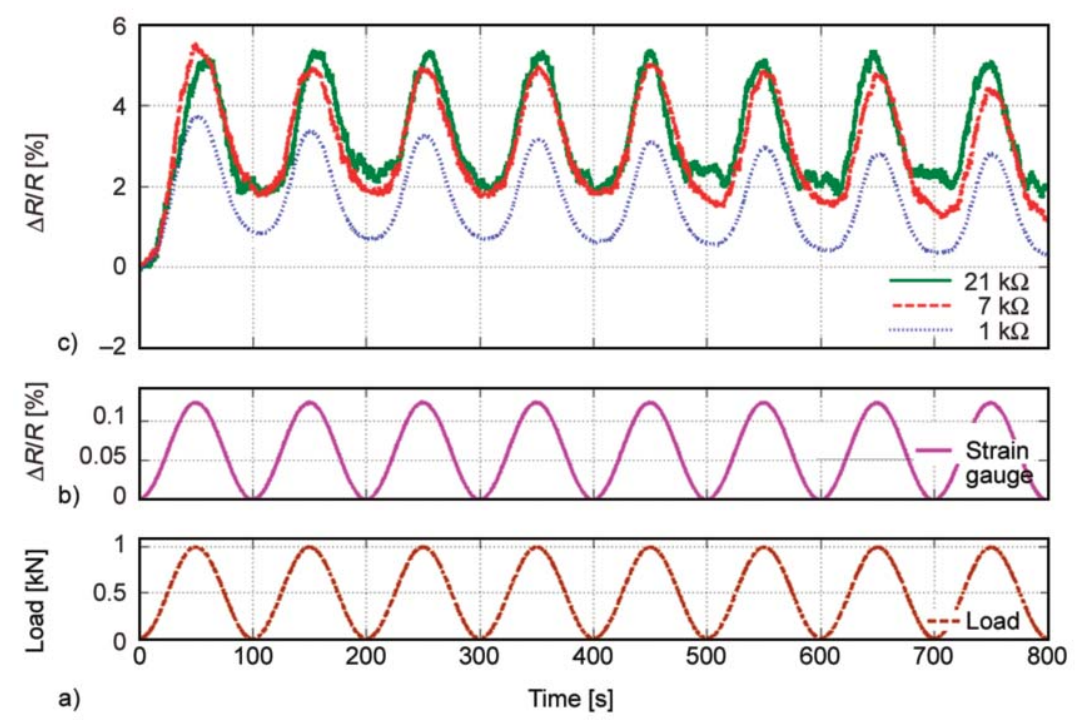

Figure 7. a) load on the GFRP specimen vs time b) change in the resistance of strain gauge and c) normalized resistance vs time for cyclic loading (tension-tension) at load control of $1 \mathrm{kN}$.

and the simple process to tailor the sensitivity demonstrates that the GNP spray coated sensor can be a potential candidate for structural health monitoring of the polymer matrix composite structures.

\section{References}

[1] Soma Sekhar B. V., Balasubramaniam K, Krishnamurthy C. V.: Structural health monitoring of fiber-reinforced composite plates for low-velocity impact damage using ultrasonic lamb wave tomography. Structural Health Monitoring, 5, 243-253 (2016). https://doi.org/10.1177/1475921706067739

[2] Garcia I., Zubia J., Durana G., Aldabaldetreku G., Illarramendi M. A., Villatoro J.: Optical fiber sensors for aircraft structural health monitoring. Sensors, 15, 15494-15519 (2015). https://doi.org/10.3390/s150715494

[3] Stankovich S., Dikin D. A., Dornmett G. H., Kohlhaas K. M., Zimney E. J., Stach E. A., Piner R. D., Nguyen S. T., Ruoff R. S.: Graphene-based composite materials. Nature, 442, 282-286 (2006).

https://doi.org/10.1038/nature04969

[4] Oskouyi A. B., Sundararaj U., Mertiny P.: Tunneling conductivity and piezoresistivity of composites containing randomly dispersed conductive nano-platelets. Materials, 7, 2501-2521 (2014). https://doi.org/10.3390/ma7042501

[5] Miller S. G., Baue J. L., Maryanski M. J., Heimann P. J., Barlow J. P., Gosau J. M., Allred R. E.: Characterization of epoxy functionalized graphite nanoparticles and the physical properties of epoxy matrix nanocomposites. Composites Science and Technology, 70, 1120-1125 (2010).

https://doi.org/10.1016/j.compscitech.2010.02.023
[6] Wu L. L., Lv X., Zhang C. C.: Effect of modified graphene addition on the electrical properties of epoxy resin composite. Advanced Materials Research, 239242, 55-58 (2011).

https://doi.org/10.4028/www.scientific.net/AMR.239-242.55

[7] Qu S., Wong S-C.: Piezoresistive behavior of polymer reinforced by expanded graphite. Composites Science and Technology, 67, 231-237 (2007).

https://doi.org/10.1016/j.compscitech.2006.08.008

[8] Doll J. C., Pruitt B. L.: Design of piezoresistive versus piezoelectric contact mode scanning probes. Journal of Micromechanics and Microengineering, 20, 095023/1095023/15 (2010).

https://doi.org/10.1088/0960-1317/20/9/095023

[9] Meric I., Han M. Y., Young A. F., Ozyilmaz B., Kim P., Shepard K. L.: Current saturation in zero-bandgap, topgated graphene field-effect transistors. Nature Nanotechnology, 3, 654-659 (2008).

https://doi.org/10.1038/nnano.2008.268

[10] Balandin A. A., Ghosh S., Bao W., Calizo I., Teweldebrhan D., Miao F., Lau C. N.: Superior thermal conductivity of single-layer graphene. Nano Letters, 8, 902907 (2008).

https://doi.org/10.1021/n10731872

[11] Lee C., Wei X., Kysar J. W., Hone J.: Measurement of the elastic properties and intrinsic strength of monolayer graphene. Science, 321, 385-388 (2008). https://doi.org/10.1126/science.1157996

[12] Kim B. J., Jang H., Lee S-K., Hong B. H., Ahn J-H., Cho J. H.: High-performance flexible graphene field effect transistors with ion gel gate dielectrics. Nano Letters, 10, 3464-3466 (2010). https://doi.org/10.1021/nl101559n

[13] Hill E. W., Vijayaragahvan A., Novoselov K.: Graphene sensors. IEEE Sensors Journal, 11, 3161-3170 (2011). https://doi.org/10.1109/JSEN.2011.2167608 
[14] Zhao J., He C., Yang R., Shi Z., Cheng M., Yang W., Xie G., Wang D., Shi D., Zhang G.: Ultra-sensitive strain sensors based on piezoresistive nanographene films. Applied Physics Letter, 101, 063112/1-063112/5 (2012). https://doi.org/10.1063/1.4742331

[15] Eswaraiah V., Balasubramaniam K., Ramaprabhu S.: Functionalized graphene reinforced thermoplastic nanocomposites as strain sensors in structural health monitoring. Journal of Materials Chemistry, 21, 12626-12628 (2011). https://doi.org/10.1039/C1JM12302E

[16] Wang Y., Yang R., Shi Z., Zhang L., Shi D., Wang E., Zhang G.: Super-elastic graphene ripples for flexible strain sensors. ACS Nano, 5, 3645-3650 (2011). https://doi.org/10.1021/nn103523t

[17] Lee Y., Bae S., Jang H., Jang S., Zhu S-E., Sim S. H., Song Y. I., Hong B. H., Ahn J-H.: Wafer-scale synthesis and transfer of graphene films. Nano Letters, 10, 490493 (2010).

https://doi.org/10.1021/n1903272n

[18] Makireddi S., Varghese F. V., Balasubramaniam K.: Non-monotonic piezoresistive behaviour of graphene nanoplatelet (GNP)-polymer composite flexible films prepared by solvent casting. Express Polymer Letters, 11, 581-588 (2017). https://doi.org/10.3144/expresspolymlett.2017.55

[19] Zhao J., Wang G., Yang R., Lu X., Cheng M., He C., Xie G., Meng J., Shi D., Zhang G.: Tunable piezoresistivity of nanographene films for strain sensing. ACS Nano, 9, 1622-1629 (2015). https://doi.org/10.1021/nn506341u

[20] Hempel M., Nezich D., Kong J., Hofmann M.: A novel class of strain gauges based on layered percolative films of 2D materials. Nano Letters, 12, 5714-5718 (2012). https://doi.org/10.1021/nl302959a

[21] Wang D-Y., Tao L-Q., Liu Y., Zhang T-Y., Pang Y., Wang Q., Jiang S., Yang Y., Ren T-L.: High performance flexible strain sensor based on self-locked overlapping graphene sheets. Nanoscale, 8, 20090-20095 (2016). https://doi.org/10.1039/C6NR07620C
[22] Chiacchiarelli L. M., Rallini M., Monti M., Puglia D., Kenny J. M., Torre L.: The role of irreversible and reversible phenomena in the piezoresistive behavior of graphene epoxy nanocomposites applied to structural health monitoring. Composites Science and Technology, 80, 73-79 (2013).

https://doi.org/10.1016/j.compscitech.2013.03.009

[23] Tamburrano A., Sarasini F., de Bellis G., D’Aloia A. G., Sarto M. S.: The piezoresistive effect in graphene-based polymeric composite. Nanotechnology, 24, 465702/1465702/10 (2013).

https://doi.org/10.1088/0957-4484/24/46/465702

[24] Rinaldi A., Proietti A., Tamburrano A., Ciminello M., Sarto M. S.: Graphene-based strain sensor array on carbon fiber composite laminate. IEEE Sensors Journal, 15, 7295-7303 (2015). https://doi.org/10.1109/JSEN.2015.2472595

[25] Zhang X-W., Pan Y., Zheng Q., Yi X-S.: Time dependence of piezoresistance for the conductor-filled polymer composites. Journal Polymer Science Part B: Polymer Physics, 38, 2739-2749 (2000).

https://doi.org/10.1002/10990488(20001101)38:21<2739::AID-POLB40>3.0.CO;2-O

[26] Hu N., Karube Y., Yan C., Masuda Z., Fukunaga H.: Tunneling effect in a polymer/carbon nanotube nanocomposite strain sensor. Acta Materialia, 56, 29292936 (2008).

https://doi.org/10.1016/j.actamat.2008.02.030

[27] Amjadi M., Yoon Y. J., Park I.: Ultra-stretchable and skin-mountable strain sensors using carbon nanotubesEcoflex nanocomposites. Nanotechnology, 26, 375501/1375501/11 (2015). https://doi.org/10.1088/0957-4484/26/37/375501

[28] Sai M., Sethy D., Varghese F. V., Balasubramaniam K.: Cyclic loading behaviour and crack monitoring potential of graphene nanoplatelet (GNP) based strain sensors in simple structures. Material Research Express, 5, 035701/1-35701/9 (2018). https://doi.org/10.1088/2053-1591/aaaf7b

[29] Hu Y., Zhao T., Zhu P., Zhang Y., Liang X., Sun R., Wong C-P.: A low-cost, printable, and stretchable strain sensor based on highly conductive elastic composites with tunable sensitivity for human motion monitoring. Nano Research, 11, 1938-1955 (2018). https://doi.org/10.1007/s12274-017-1811-0 